\title{
Holistic Urban Seismic Risk Evaluation of Megacities: Application and Robustness
}

\author{
Martha Liliana Carreño, Omar D. Cardona, Mabel-Cristina Marulanda and \\ Alex H. Barbat
}

\section{Risk from a Holistic Perspective}

Disaster risk has been defined, for management purposes, as the potential economic, social and environmental consequences of hazardous events that may occur in a specified period of time. However, in the past, the concept of risk has been defined in a fragmentary way in many cases, according to each scientific discipline involved in its appraisal (Cardona, 2004). Based on the formulation of disaster risk of UNDRO (1980) several methodologies for risk assessment have been developed from different perspectives in the last decades. From a holistic perspective, disaster risk requires a multidisciplinary evaluation that takes into account not only the expected physical damage, the number and type of casualties or economic losses (direct impact), but also the conditions related to social fragility and lack of resilience conditions, which favour the second order effects (indirect impact) when a hazard event strike an urban centre (Cardona and Hurtado, 2000; Masure, 2003; Carreño et al., 2007a).

Cardona (2001) developed a conceptual framework and a model for disaster risk analysis of a city from a holistic perspective. It considers both "hard" and "soft" risk variables of the urban centre, taking into account exposure, socioeconomic characteristics of the different localities (units) of the city and their disaster coping capacity or degree of resilience. The model was made to guide the decision-making in risk management, helping to identify the critical zones of the city and their vulnerability from different professional disciplines. Carreño (2006) developed an alternative method for Urban Risk Evaluation, based on Cardona's model (Cardona, 2001; Barbat and Cardona, 2003). The urban risk is evaluated using composite indicators or indices. Expected building damage and losses in the infrastructure, obtained from future loss scenarios are basic information for the evaluation of a physical risk index in each unit of analysis.

M.-L. Carreño ( $\square)$

Technical University of Catalonia. Campus Norte. C/ Gran Capitán sn, Mod. C1, 08034, Barcelona, Spain

e-mail: liliana@cimne.upc.edu 
Often, when historical information is available, the principal hazard can be usually identified and thus the most potential critical situation for the city.

The holistic evaluation of disaster risk is achieved affecting the physical risk with an impact factor, obtained from contextual conditions, such as the socioeconomic fragility and the lack of resilience, that aggravate initial physical loss scenario. Available data about these conditions at urban level are necessary to apply the method. A brief explanation of the model is made forward to illustrate the benefits of this approach that contributes to the effectiveness of disaster risk management, inviting to the action identifying the hard and soft weaknesses of the urban centre. Figure 1 shows the theoretical framework of the alternative model.

From a holistic perspective risk, $R$, is a function of the potential physical damage, $D_{j}$, and an aggravating coefficient, $I_{f}$. The former is obtained from the susceptibility of the exposed elements, $\gamma_{D i}$, to hazards, $H_{i}$, regarding their potential intensities, $I$, of events in a period of time $t$, and the latter depends on the social fragilities, $\gamma_{F i}$, and the issues related to lack of resilience, $\gamma_{R i}$, of the disaster prone socio-technical system or context. Using the meta-concepts of the theory of control and complex system dynamics to reduce risk, it is necessary to intervene in corrective and prospective way the vulnerability factors and, when it is possible, the hazards directly. Then risk management requires a
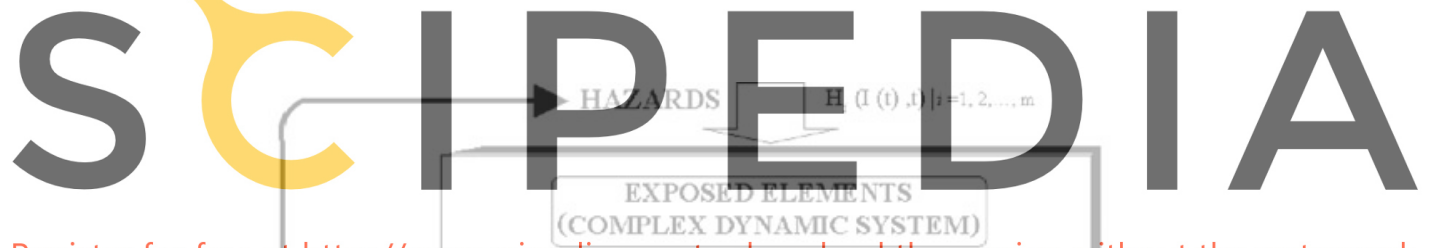

Register for free at https//www.scipedia.com to download the version without the watermark

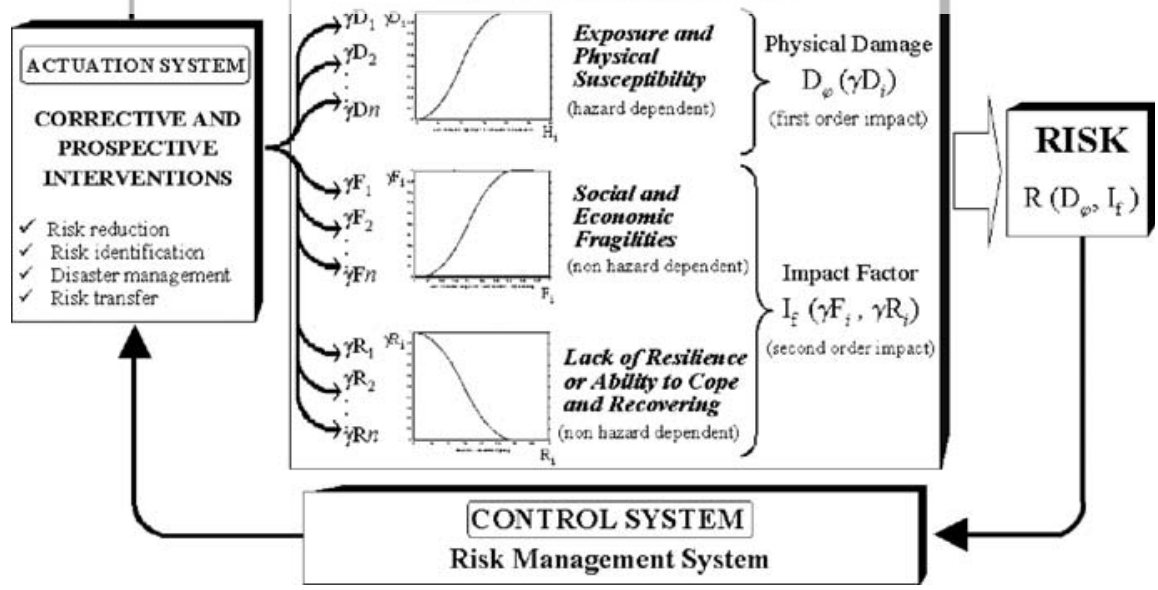

Fig. 1 Theoretical framework and model for holistic approach of disaster risk (adapted from Cardona and Barbat, 2000) 
system of control (institutional structure) and an actuation system (public policies and actions) to implement the changes needed on the exposed elements or complex system where risk is a social process.

\subsection{The Urban Seismic Risk Index - USRi}

The USRi is a composite indicator -or index-that measures seismic risk from an integrated and comprehensive perspective and guides decision-making identifying the main multidisciplinary factors of vulnerability to be reduced or intervened. The first step of the method is the evaluation of the potential physical damage (hard approach) as the result of the convolution of seismic hazard and physical vulnerability of buildings and infrastructure. Subsequently, a set of social context conditions that aggravate the physical effects are also considered (soft approach). According to this procedure, a physical risk index is obtained, for each unit of analysis, from existing loss scenarios, whereas the total risk index is obtained by factoring the former index by an impact factor using an aggravating coefficient, based on variables associated with the socioeconomic conditions of each unit of analysis.

The proposed holistic evaluation of risk is performed using a set of input

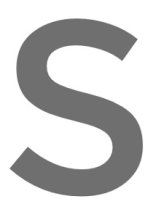
variables, herein d aggravating conditior tors are obtained capacity informatio The USRi d
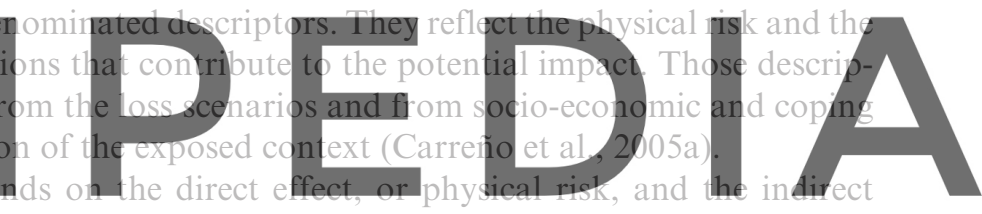

effects expressed as a factor of the direct effects. Therefore, the USRi for each

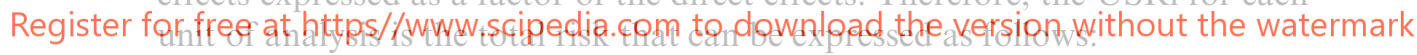

$$
U S R i=R_{T}=R_{F}(1+F)
$$

expression known as the Moncho's Equation in the field of disaster risk indicators, where $R_{T}$ is the total risk index, $R_{F}$ is the physical risk index and the indirect effects given by an impact factor $(1+F)$, based on an aggravating coefficient, $F$ is the aggravating coefficient.

The physical risk index, $R_{F}$, depends on the weighted sum of a set of component factors

$$
R_{F}=\sum_{i=1}^{p} F_{R F i} w_{R F i}
$$

where $p$ is the total number of descriptors of physical risk index, $F_{R F i}$ are the component factors and $w_{R F i}$ are their weights respectively. The factors of physical risk, $F_{R F i}$, are based on the gross values of physical risk descriptors such as the number of deaths, injured or the destroyed area, and so on. It has to be mentioned that the calculation of physical risk scenarios is not the objective 
of the methodology developed in this chapter, but the physical risk index is obtained starting from existing loss evaluations.

The coefficient, $F$, is another index evaluated in the same way. It depends on the weighted sum of a set of aggravating factors related to the socio-economic fragility, $F_{F S i}$, and the lack of resilience of the exposed context, $F_{F R j}$

$$
F=\sum_{i=1}^{m} F_{F S i} w_{F S i}+\sum_{j=1}^{m} F_{F R j} w_{F R j}
$$

where $w_{F S i}$ and $w_{F R j}$ are the weights or influences of each $i$ and $j$ factors and $m$ and $n$ are the total number of descriptors for social fragility and lack of resilience respectively. Figure 2 shows the process of calculation of the total risk index for the geographic units of analysis, which could be districts, municipalities, communes or localities. See Carreño et al. (2007a) for measurement units of each descriptor and a detailed explanation of the method.

It is estimated that the indirect effects of hazard events, sized by the coefficient $F$ in Equation (1), can be of the same order than the direct effects. According to the Economic Commission for Latin America and the Caribbean
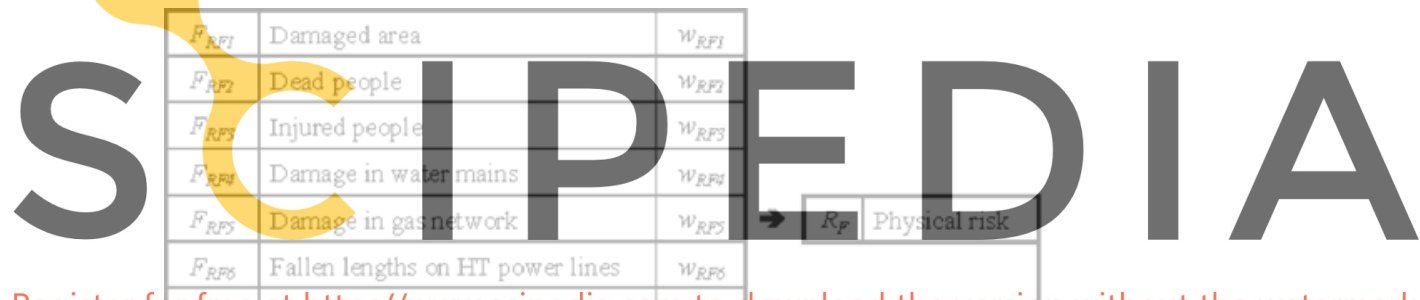

Register for free at hattps KAwaww.scipedia.com, do download the version without the watermark

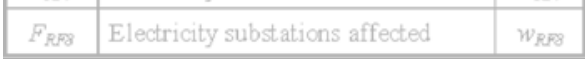

\begin{tabular}{|c|c|c|c|c|}
\hline$F_{F S I}$ & Slums-squatter neighbourhoods & $w_{P S I}$ & & \\
\hline$F_{F S Z}$ & Mortality rate & $w_{P S Z}$ & & \\
\hline$F_{F S 3}$ & Delinquency rate & $w_{P S S}$ & & \\
\hline$F_{F S 4}$ & Social disparity index & $w_{P S A}$ & & \\
\hline$F_{\text {FSS }}$ & Population density & $w_{P S S}$ & & \\
\hline$F_{F R I}$ & Hospital beds & $w_{P R I}$ & $F$ & Impact factor \\
\hline$F_{F R Z}$ & Health human resources & $w_{F R 2}$ & & \\
\hline$F_{F R 3}$ & Public space & $w_{F R 3}$ & & \\
\hline$F_{F R}$ & Rescue and firemen manpower & $w_{F R 4}$ & & \\
\hline$F_{\overline{F T S}}$ & Dev elopment level & $w_{P P S}$ & & \\
\hline$F_{F \overline{R S}}$ & Emergency planning & $w_{F R \sigma}$ & & \\
\hline
\end{tabular}

\begin{tabular}{|l|l|l|l|l|l|l}
\hline$R_{T}$ & Total risk \\
\hline
\end{tabular}

Fig. 2 Factors of physical risk, social fragility and lack of resilience and their weights 
(Zapata, 2004), it is estimated that the indirect economic effects of a natural disaster depend on the type of phenomenon. The order of magnitude of the indirect economic effects for a "wet" disaster (as one caused by a flood) could be of $0.50-0.75$ of the direct effects. In the case of a "dry" disaster (caused by an earthquake, for example), the indirect effects could be about the $0.75-1.00$ of the direct effects, due to the kind of damage (destruction of livelihoods, infrastructure, housing, etc.). This means that the total risk, $R_{T}$, could be between 1.5 and 2 times $R_{F}$. In this method, the maximum value selected was the latter. For this reason, the aggravating coefficient, $F$, takes values between 0 and 1 in Equation (1).

The factors of physical risk, $F_{R F i}$, and the aggravating factors $F_{F S i}$ and $F_{F R j}$ are calculated using transformation functions as the shown in the Fig. 3. These examples of transformation functions for physical risk, social fragility and lack of resilience (or coping capacity) show the values of the descriptors in the $x$-axis and the corresponding factors, or scaled values, in the $y$-axis.

These functions describe the intensity of the risk for each descriptor and standardise the gross values of the descriptors transforming them in commensurable factors. Sigmoid functions were used in most of the cases to develop the transformation functions. All their maximum and minimum values (corresponding to the values 1.0 or 0.0 of each factor) were fixed using existing information about past disasters as well as the expert opinion. For example the transformation (0.0) when this desc ip for a potential dam The weights

factor and are calculated by means of the Analytic Hierarchy Process (AHP), Register for free at https//www.scipedia.com to download the version without the watermark

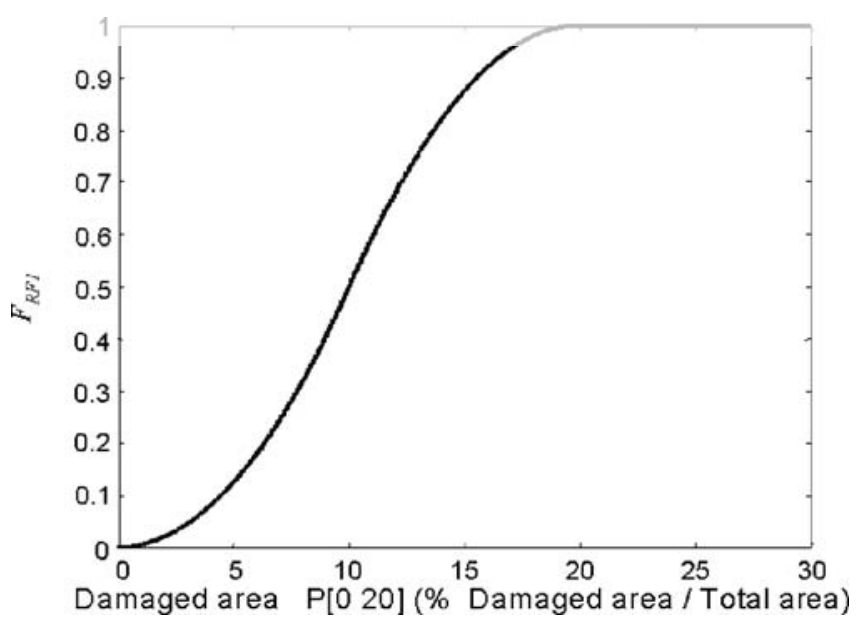

Fig. 3 Example of transformation functions used to standardise physical risk, social fragility and lack of resilience factors 
which is used to derive ratio scales from both discrete and continuous paired comparisons (Saaty, 2001). This process has been performed starting from experts opinions collected by means of the Delphi method. This is the most adequate way of judging the relative importance of variables having different nature and calculating their relative weights.

\subsection{Improvement of the Previous Approach}

The model of holistic urban risk evaluation proposed Carreño et al. (2007a,b) improves conceptual and methodological aspects of the first proposal of Cardona (2001), refining the applied numerical techniques and turning it into a more versatile tool. The conceptual improvements provide a more solid theoretical and analytical support to the new model, eliminating unnecessary and dubious aspects of the previous method and giving more transparency and applicability in some cases. Cardona's model allows the evaluation of the seismic risk in an urban center taking into account the characteristics of the physical risk, seismic hazard, physical exposure, socio-economical fragility and lack of resilience, what permits to identify those characteristics of the city that increase the level of risk and also the critical areas. This model studies different types of information by means of indicators and use on the standard dev the results obtaind seismic risk among the different atean comparison in absolute terms with other urban
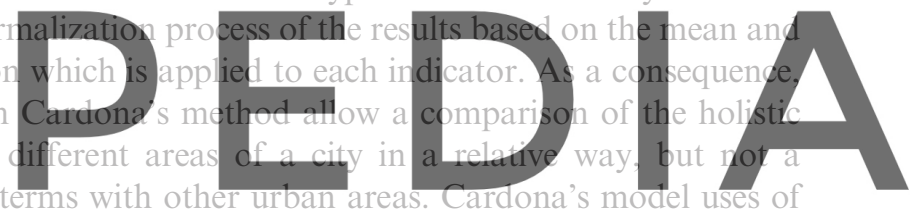

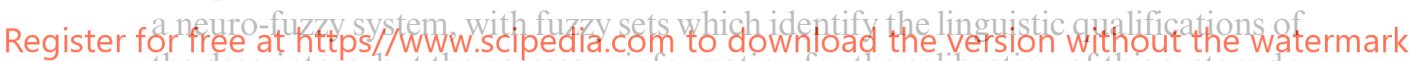
the descriptors, but the necessary information for the calibration of this system do not exist

The new method proposed in this chapter conserves the approach based on indicators, but it improves the procedure of normalization and calculates the final indices in an absolute (non relative) manner. This feature facilitates the comparison of risk among urban centers. The exposure and the seismic hazard have been eliminated in the method proposed in this chapter because they have been included into the physical risk variables calculation. The descriptor of population density, a component of the exposure in Cardona's model, is now included as a descriptor of social fragility. The new approach preserves the use of indicators and fuzzy sets or membership functions, proposed originally by Cardona, but in a different way. Other improvements of the proposed model refer to the units of some of the descriptors; in certain cases it is more important to normalize the input values respecting the population than with respect of the area of the studied zone. This is, for example, the case of the number of hospital beds existing in the studied urban area. The socio-economic fragility and the lack of resilience are a set of factors (related to indirect or intangible effects) that aggravate the physical risk (potential direct effects). 


\section{Application to Megacities}

The Urban Seismic Risk Index was proposed and applied to Barcelona (Spain) and Bogota (Colombia) by Carreño (2006) and by Carreño et al. (2005b, 2007a) and by Suarez (2007) to Manizales (Colombia). Recently, the USRi has been applied to Metro Manila (The Philippines) by Earthquake Megacities Initiative (EMI, 2006). In the case of Bogota the scenarios of losses building by building were developed by Universidad de Los Andes (2005). For the city of Barcelona probabilistic and deterministic scenarios were developed by ICC/CIMNE (2004). In Manizales the damage scenarios were made by ERN (2005). This section presents the summary of the USRi results for the all cities of Metro Manila to illustrate its application to other metropolitan urban centre.

\subsection{Results for Metro-Manila}

Metropolitan Manila, the capital city the Philippines is officially called the National Capital Region (NCR). Although it is the smallest region, it is the most densely populated region of the country. Metro Manila is composed by 4 municipalities and 13 cities thereof into an integrated unit with the manager or

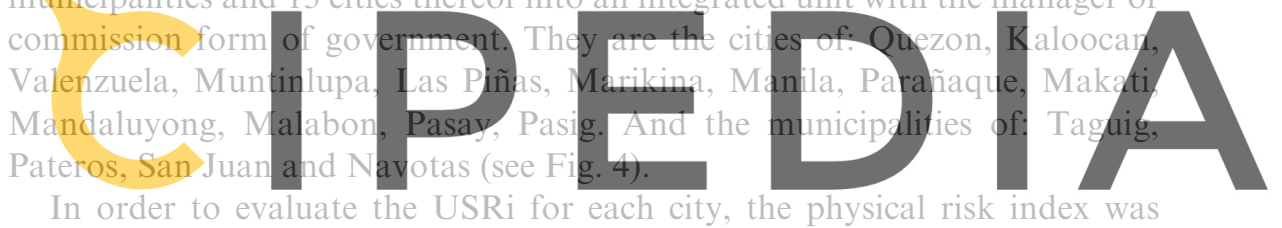

In order to evaluate the USRi for each city, the physical risk index was

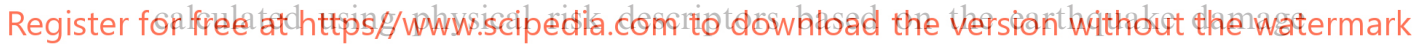

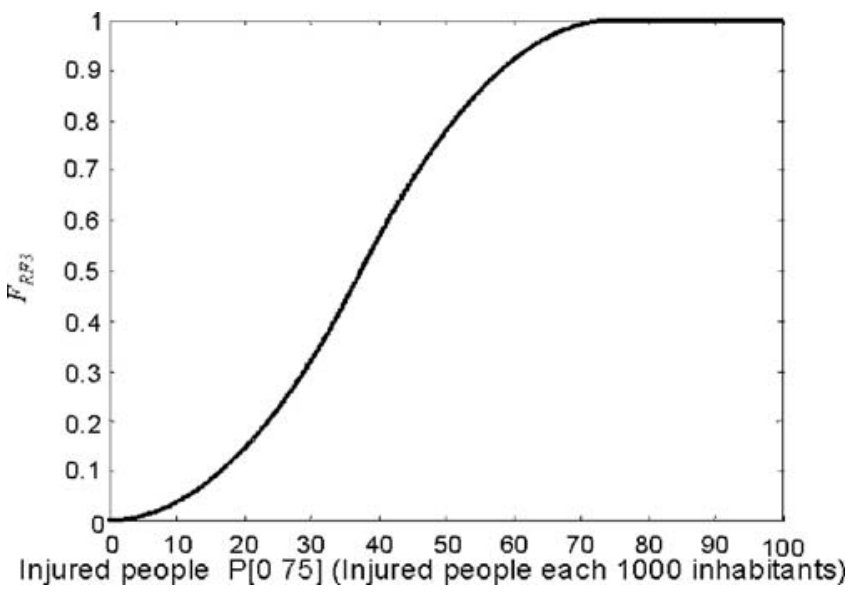

Fig. 4 Territorial division of Metro Manila, Philippines 


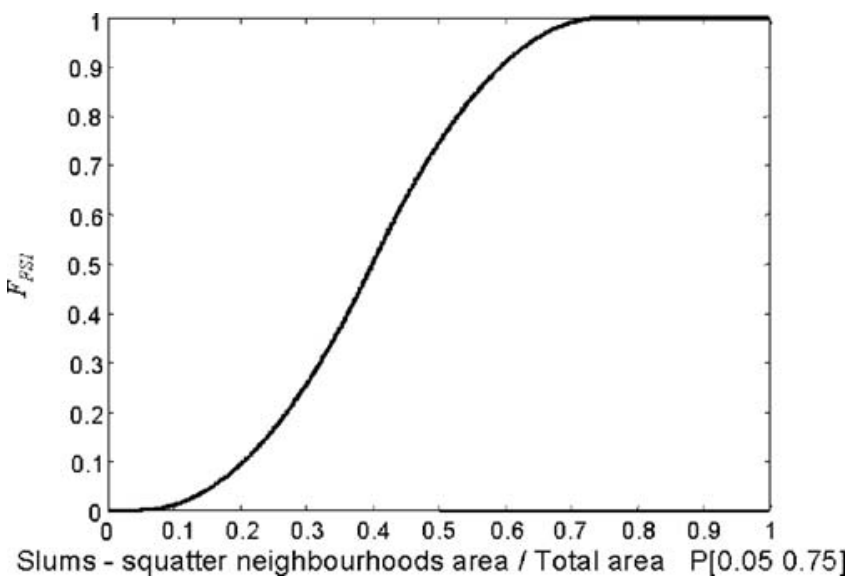

Fig. 5 Physical risk index for Metro Manila

MMEIRS-08, obtained from the Earthquake Impact Reduction Study of Metro Manila (MMEIRS). This scenario corresponds to an earthquake of Magnitude 7.2, in the West Valley Fault, with $2 \mathrm{~km}$ of depth. Figures 5, 6, 7 show the results for the physical risk index, the aggravating coefficient and
the total risk index (USRi) for Metro Manila using the model above
described.
Cities in Metro Manila were clustered according to their leyel of risk in four
different arrays aceording to the total USRi and its components, phy sical risk and aggravating factor (social fragility + lack of resilience), this grouping is Register forf free at htttis / /Www.scipedia.com to download the version without the watermark

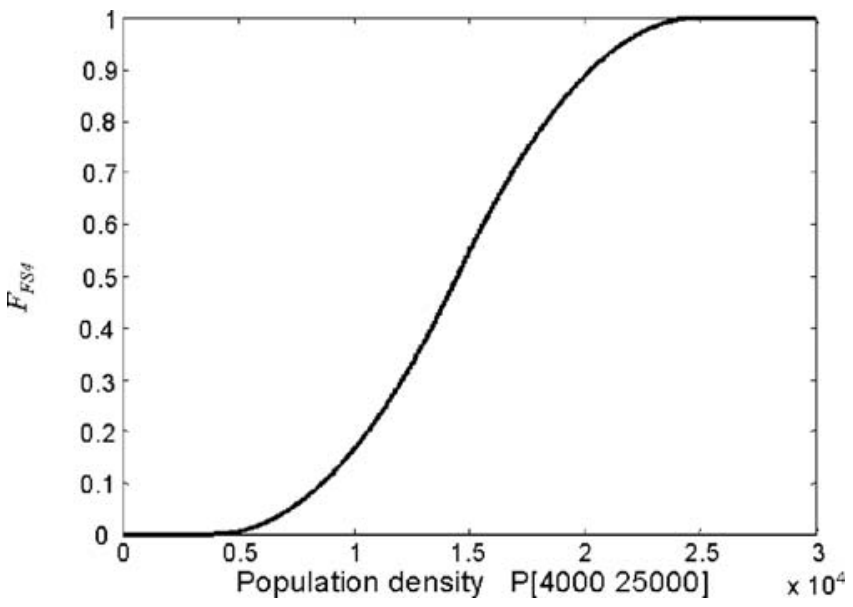

Fig. 6 Aggravating coefficient for Metro Manila 


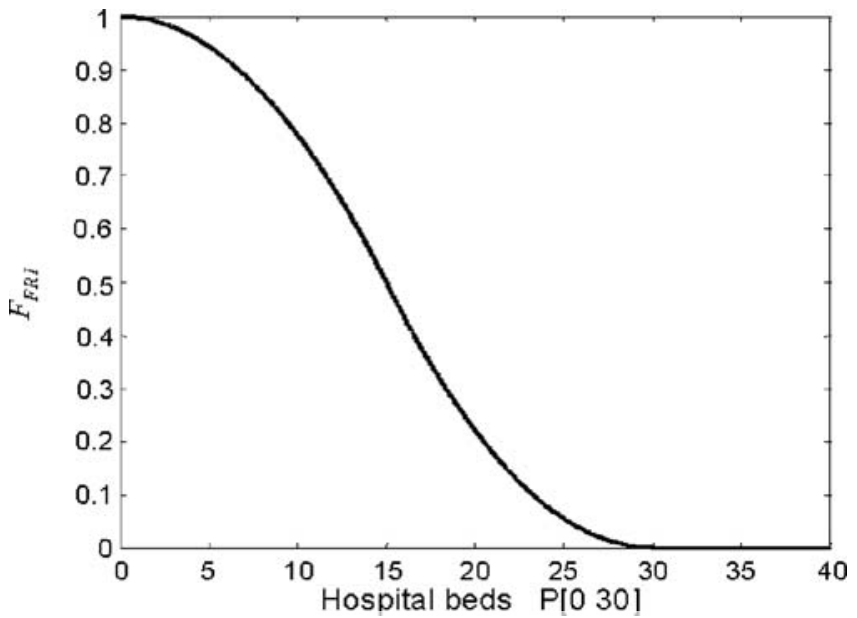

Fig. 7 Total risk index for Metro Manila

Table 1 Holistic urban seismic risk of Metro Manila

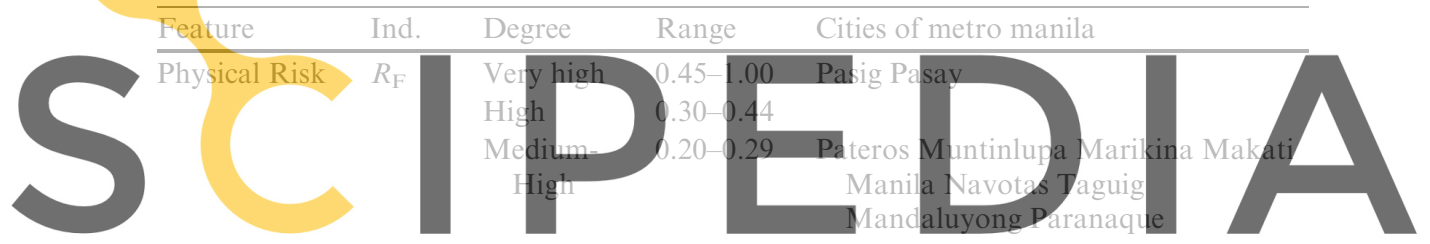

Medium- $\quad$ 0.10-0.19 Las Piñas Quezon Malabon San Juan

Register for free at https//www.scipedia.com to download the version without the watermark

Low 0.00-0.09 Valenzuela Kalookan

\begin{tabular}{|c|c|c|c|c|}
\hline \multirow{5}{*}{$\begin{array}{l}\text { Aggravating } \\
\text { Coefficient }\end{array}$} & \multirow[t]{5}{*}{$F$} & Very High & $0.65-1.00$ & Navotas Malabon Taguig San Juan \\
\hline & & High & $0.55-0.64$ & $\begin{array}{l}\text { Kalookan Valenzuela Pasay Pateros } \\
\text { Las Piñas Quezon Pasig }\end{array}$ \\
\hline & & $\begin{array}{l}\text { Medium- } \\
\text { High }\end{array}$ & $0.40-0.54$ & $\begin{array}{l}\text { Marikina Paranaque Mandaluyong } \\
\text { Manila Makati Muntinlupa }\end{array}$ \\
\hline & & $\begin{array}{l}\text { Medium- } \\
\text { Low }\end{array}$ & $0.20-0.39$ & \\
\hline & & Low & $0.00-0.19$ & \\
\hline \multirow[t]{5}{*}{ Total Risk } & \multirow[t]{5}{*}{ USRi } & Very High & $0.70-1.00$ & Pasay Pasig \\
\hline & & High & $0.45-0.69$ & Navotas Pateros Marikina Taguig \\
\hline & & $\begin{array}{l}\text { Medium- } \\
\text { High }\end{array}$ & $0.30-0.44$ & $\begin{array}{l}\text { Muntinlupa Manila Makati } \\
\text { Mandaluyong Paranaque }\end{array}$ \\
\hline & & $\begin{array}{l}\text { Medium- } \\
\text { Low }\end{array}$ & $0.15-0.29$ & Las Piñas Quezon Malabon San Juan \\
\hline & & Low & $0.00-0.14$ & Kalookan Velenzuela \\
\hline
\end{tabular}




\subsection{Sensitivity Analysis}

Sensitivity analysis is also referred to as robustness analysis of the model. In this case, the sensitivity analysis studies how the variation in the values of the index of Total Risk, $R_{T}$, can be apportioned, qualitatively or quantitatively, to different sources of variation, and how this given composite indicator, depends upon the information fed into it. In other words, once the proposed model (composite indicator) is determined, it is important to analyse how much the results are influenced by uncertainty in the source data or uncertainty in the weights and transformation functions, due to the stakeholders' subjectivity or plurality of perspectives. For this analysis a Monte Carlo-based simulation was performed.

The Monte Carlo method is a technique that uses sets of random numbers as input parameters and probability distributions for iteratively evaluating a deterministic model. This method is often used when the model is complex, non-linear or involves more than just a couple uncertain parameters. The Monte Carlo simulation is one of many methods for analysing uncertainty propagation, where the goal is to determine how random variation affects the sensitivity, performance or reliability of the model. In this case, the values of the $R_{T}$ for each territorial unit were obtained five thousand times, using random

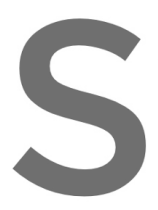
sets of imput data, transformation functions and we
within an acceptable range of variability.
The random values, for each set previously ment
vidually by using uniform distributions in each in
random values for simultaneous variation of the a neous variatic
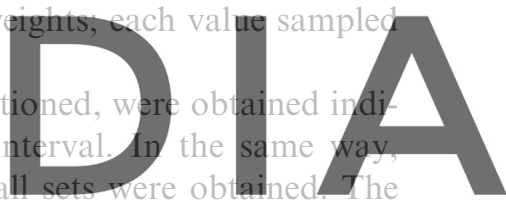

minimum and maximum values were chosen according to expert opinions to Register fok free at httes/fwww.scipedias.som to downdgad the version withouth the watermark

of stochastic results were created with random inputs of each parameter (input data, transformation functions, weights and all simultaneously) for each territorial unit of analysis. In order to provide a concise summary of the results, the mean, median, standard deviation, maximum and minimum values and a few other summary statistics to describe the resulting distribution were reported. Likewise, a histogram was created for visualizing the uncertainty of results, which illustrates the profile of results, the uncertainty degree and the existing distribution. In addition, the cumulative distribution function was included in each graph to illustrate the percentage of data points that are below a value or point of interest.

Figure 8 shows an example of a histogram and Table 2 illustrates an example of a statistic summary of the Monte Carlo simulation.

Once the results were calculated through Monte Carlo procedure, the variability or volatility graphics were built to compare the stochastic results of $R_{T}$ to the fixed results obtained from the methodology. Figures 9 and 10 illustrate minimum $(\triangle)$, maximum $(\times)$ and mean $(\bullet)$ values and the bars represent the fixed values obtained for each territorial unit. It can be seen that for the 


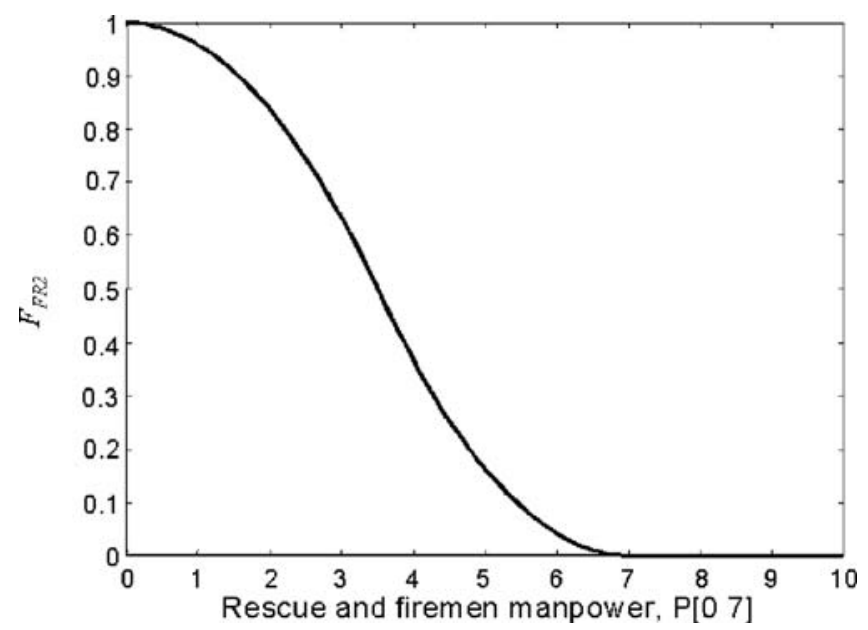

Fig. 8 Example of a histogram of the results of RT for Mandaluyong City with stochastic weights

variability of each parameter the volatility of values is not so big. The uncertainty is bigger in the case of the simultaneous variation of all parameters.

Table 3 shows that the results obtained through the simulation are very similar to the results obtained using fixed values of input data, weights and transformation functions of the method previously described and applied to Metro Manila. The overall results show that the cities of the metropolitan area vary slightly in their rankings. Some units fluctuate at the most by one position. In other urban centres, as Barcelona and Manizales, where the method has been applied and where a similar sensitivity analysis has been made, the results are similar than in Metro Manila. Only in the case of

Table 2 Example of statistic summary of the results for Mandaluyong City with stochastic weights

\begin{tabular}{|c|c|c|c|c|c|c|c|}
\hline \multicolumn{3}{|c|}{ Central tendency (location) } & & \multicolumn{4}{|c|}{ Quantiles, percentiles, intervals } \\
\hline \multicolumn{2}{|c|}{ Sample Size (N): } & \multirow{3}{*}{$\begin{array}{l}5000 \\
\text { Median }\end{array}$} & & \multicolumn{2}{|l|}{$90 \%$ Interval } & \multicolumn{2}{|c|}{$95 \%$ Interval } \\
\hline & & & & $\mathrm{Q}(.05):$ & 0.343 & $\mathrm{Q}(.025):$ & 0.34 \\
\hline Mean & 0.40 & & 0.40 & $\mathrm{Q}(.95):$ & 0.459 & Q(.975): & 0.47 \\
\hline StErr: & 0.00 & & & & & & \\
\hline Skewness & 0.0435 & & & Alpha (a): & 0.05 & $\mathrm{Q}(\mathrm{a} / 2):$ & 0.34 \\
\hline Kurtosis & -1.0225 & & & $\%$ Interval: & $95 \%$ & $\mathrm{Q}(1-\mathrm{a} / 2)$ & 0.47 \\
\hline \multicolumn{4}{|l|}{ Spread } & \multicolumn{4}{|l|}{ Probabilities } \\
\hline StDev & 0.04 & & & $\operatorname{Pr}(\mathbf{y}<0.3$ & & & $0.44 \%$ \\
\hline Max: & 0.49 & $\mathrm{Q}(.75):$ & 0.43 & $\operatorname{Pr}(\mathbf{y}>0.4$ & $=$ & & $49.93 \%$ \\
\hline Min: & 0.32 & $\mathrm{Q}(.25)$ : & 0.37 & $\operatorname{Pr}(0.33<y$ & $0.4)=$ & & $49.63 \%$ \\
\hline Range & 0.17 & IQ Range: & 0.06 & Alpha (a): & & & 0.5037 \\
\hline
\end{tabular}




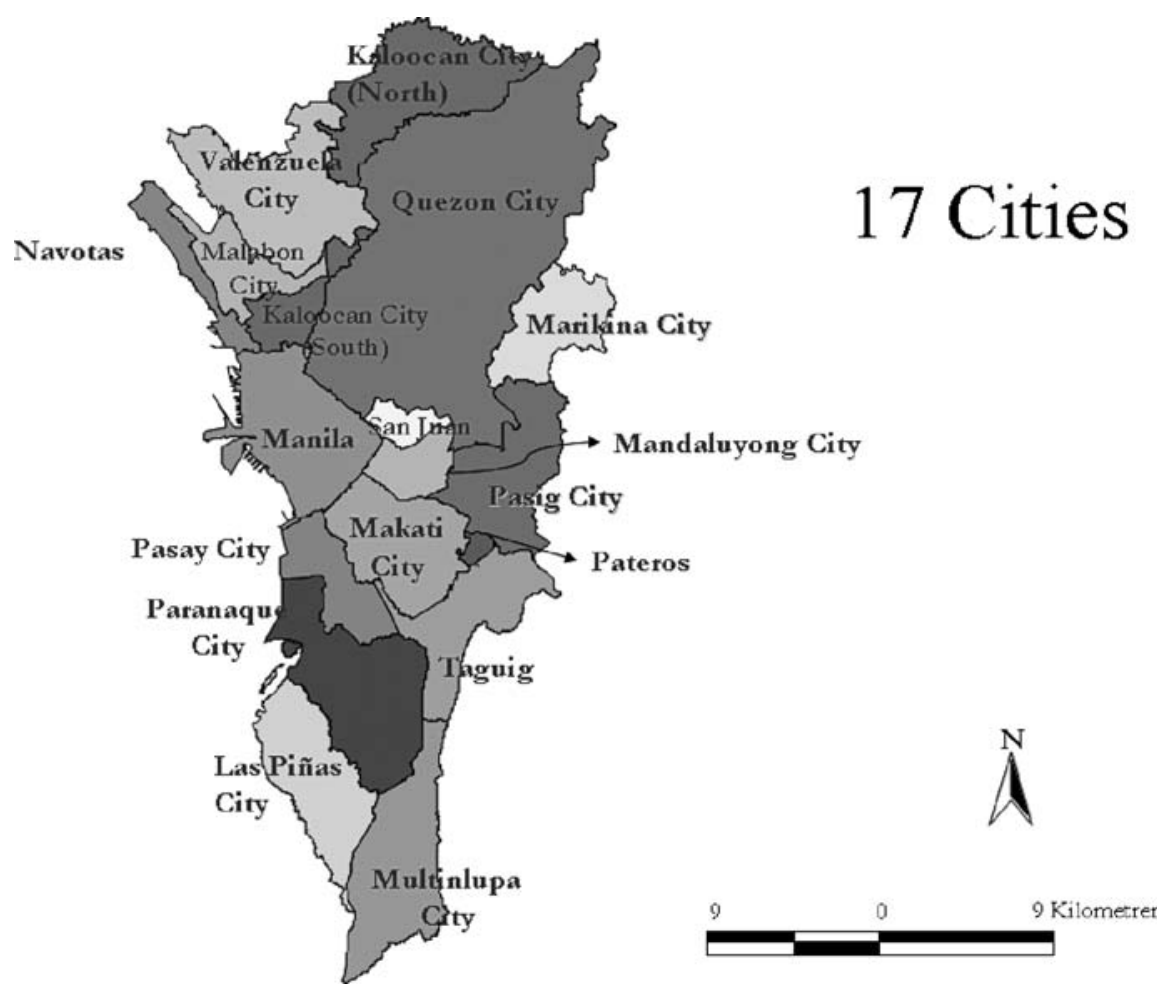

Fig. 9 Variation of the simulation results and fixed results for stochastic weights and stochastic data

Bogota some territorial units have been more volatile with position changes of two and three ranks; however it is not very relevant.

Therefore, according to the classification of the total risk by levels, all cities maintain the same level with exception of San Juan city which presents a change of range from medium-high to medium-low because the total risk result for fixed values is equal to 0.29 and for stochastic values the result was above 0.30 . Classification by ranges of risk has special interest, because it is more relevant to have into account the level of risk where a territorial unit is located than its final numerical value for risk management implications.

According to the comparison of the results of sensitivity analysis, based on Monte Carlo simulations, and the results obtained by the holistic seismic risk evaluation here described, it is possible to conclude that the methodology is robust. It is not very sensible to slight variations in the input data and small changes in the modelling parameters, such as weights and transformation functions. The results do not present important or extreme changes. If the range of variation of data and parameters is reasonable, as it is in the case of seismic risk, in general the results of the model will be stable and reliable. 


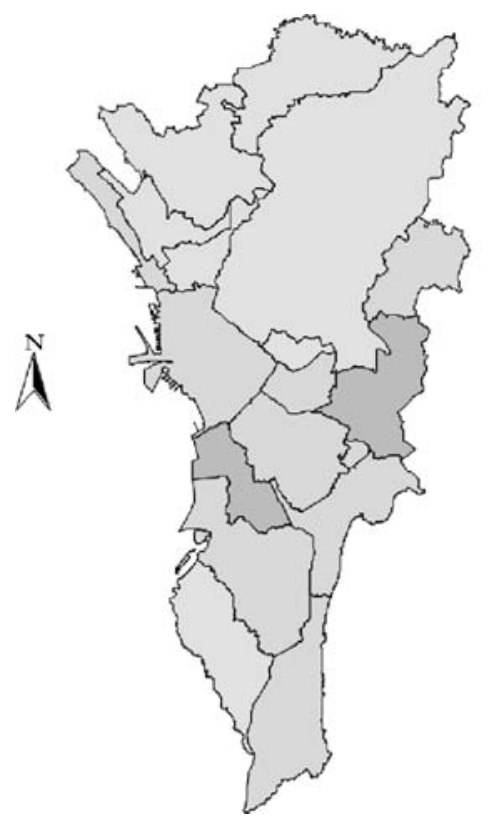

\section{Physical risk, $R_{\mathrm{F}}$}

\section{Scenario 08}

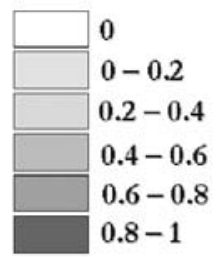

Fig. 10 Variation of the simulation results and fixed results for stochastic transformation functions and simultaneous stochastic data, weights and transformation functions

\subsection{Comparison of Results}

The results obtained for Metro Manila have been compared with those obtained for Barcelona, Bogota and Manizales. Table 4 shows the average risk values for the four cities, corresponding to the most significant scenarios in each case. Metro Manila and Bogota are located in zones with intermediate seismic hazard, whereas Barcelona is located in a zone with low to moderate seismic hazard and Manizales is placed in a zone with a high seismic hazard. The average values obtained for the physical risk index, $R_{F}$, reflect not only the seismic hazard but also the level of physical vulnerability in each city. It is interesting to remark that the results obtained for the aggravating coefficient, $F$, are not so different for the four cities. The highest value of physical risk is for Bogota, but the worst situation, taking into account the aggravating coefficient, is for Metro Manila.

\section{Conclusions}

Disaster risk estimation requires a multidisciplinary approach that takes into account not only the expected physical damage, the number and type of casualties or economic losses, but also other social, organizational and 
Table 3 Comparison between fixed values and stochastic results of RT, positions and classification of Metro Manila cities by risk levels

\begin{tabular}{|c|c|c|c|c|c|c|c|c|}
\hline \multirow[t]{2}{*}{ Level of risk } & \multicolumn{2}{|l|}{ Fixed values } & \multicolumn{2}{|l|}{ Data } & \multicolumn{2}{|l|}{ Weights } & \multicolumn{2}{|l|}{ Fuctions } \\
\hline & City & $\mathrm{R}_{\mathrm{T}}$ & City & $\mathrm{R}_{\mathrm{T}}$ & City & $\mathrm{R}_{\mathrm{T}}$ & City & $\mathrm{R}_{\mathrm{T}}$ \\
\hline \multirow[t]{2}{*}{ Very High } & Pasay & 0.72 & Pasay & 0.70 & Pasay & 0.71 & Pasay & 0.70 \\
\hline & Pasig & 0.71 & Pasig & 0.70 & Pasig & 0.70 & Pasig & 0.70 \\
\hline \multirow[t]{5}{*}{ High } & Navotas & 0.49 & Manila & 0.52 & Manila & 0.53 & Manila & 0.53 \\
\hline & Manila & 0.48 & Navotas & 0.49 & Navotas & 0.49 & Navotas & 0.49 \\
\hline & Pateros & 0.47 & Pateros & 0.49 & Pateros & 0.49 & Pateros & 0.49 \\
\hline & Marikina & 0.45 & Marikina & 0.47 & Marikina & 0.46 & Marikina & 0.47 \\
\hline & Taguig & 0.45 & Taguig & 0.46 & Taguig & 0.46 & Taguig & 0.46 \\
\hline \multirow[t]{4}{*}{ Médium-High } & Muntinlupa & 0.43 & Makati & 0.43 & Makati & 0.43 & Makati & 0.43 \\
\hline & Makati & 0.42 & Muntinlupa & 0.43 & Muntinlupa & 0.42 & Muntinlupa & 0.43 \\
\hline & Mandaluyong & 0.39 & Mandaluyong & 0.40 & Mandaluyong & 0.40 & Mandaluyong & 0.40 \\
\hline & Paranaque & 0.31 & Paranaque & 0.33 & Paranaque & 0.33 & Paranaque & 0.33 \\
\hline \multirow[t]{4}{*}{ Médium-Low } & San juan & 0.29 & San juan & 0.31 & San juan & 0.31 & San juan & 0.32 \\
\hline & Malabon & 0.26 & Malabon & 0.27 & Malabon & 0.27 & Malabon & 0.27 \\
\hline & Quezon & 0.19 & Quezon & 0.21 & Quezon & 0.21 & Quezon & 0.22 \\
\hline & Las pinas & 0.17 & Las pinas & 0.19 & Las pinas & 0.19 & Las pinas & 0.19 \\
\hline \multirow[t]{2}{*}{ Low } & Kalookan & 0.05 & Kalookan & 0.09 & Kalookan & 0.09 & Kalookan & 0.09 \\
\hline & Valenzuela & 0.03 & Valenzuela & 0.07 & Valenzuela & 0.06 & Valenzuela & 0.06 \\
\hline
\end{tabular}


Table 4 Comparison between the worst scenarios for the studied cities

\begin{tabular}{lllll}
\hline Index & Barcelona & Bogota & Manizales & Metro manila \\
\hline Physical risk, $R_{F}$ & 0.08 & 0.32 & 0.27 & 0.24 \\
Aggravating coeff. $F$ & 0.42 & 0.55 & 0.56 & 0.59 \\
USRi $=$ Total risk, $R_{T}$ & 0.11 & 0.50 & 0.44 & 0.38 \\
\hline
\end{tabular}

institutional issues related to the development of communities that contribute to the creation of risk. At the urban level, for example, vulnerability seen as an internal risk factor should be related not only to the level of exposure or the physical susceptibility of the buildings and infrastructure material elements potentially affected, but also to the social fragility and the lack of resilience or capacity to cope of the exposed community. The absence of institutional and community organization, weak preparedness for emergency response, political instability and the lack of economic health in a geographical area contribute to risk increasing. Therefore, the potential negative consequences are not only related to the effects of the hazardous event as such, but also to the capacity to absorb the effects and the control of its implications in a given geographical area.

For the modelling, a simplified but multidisciplinary representation of urban seismic risk has been suggested, based on the parametric use of variables that reflect aspects of such risk. This parametric approach is not more than a model formulated in the most realistic possible manner, to which corrections or alternative figures may be continuously introduced. The consideration of physical aspects allowed the construction of a physical risk index. Also, the contextual variables (social, economic, etc.) allowed the construction of an aggravating coefficient. The former is built from the information about the seismic scenarios of physical damage (direct effects) and the latter is the result from the estimation of aggravating conditions (indirect effects) based on descriptors and factors related to the social fragility and the lack of resilience of the exposed elements.

This new model for holistic evaluation of risk facilitates the integrated risk management by the different stakeholders involved in risk reduction decisionmaking. It permits the follow-up of the risk situation and the effectiveness of the prevention and mitigation measures can be easily achieved. Results can be verified and the mitigation priorities can be established as regards the prevention and planning actions to modify those conditions having a greater influence on risk in the city. Once the results have been expressed in graphs for each locality or district, it is easy to identify the most relevant aspects of the total risk index, with no need for further analysis and interpretation of results. Finally, this method allows to compare risk among different cities around the world and to perform a multi-hazard risk analysis.

Acknowledgements The authors express gratitude to the Inter-American Development Bank (IDB), for the financial support through the Information and Indicators Program for Disaster Risk Management for the Americas and to the ProVention Consortium for research grant to 
apply the method in Metro Manila, The authors are grateful with Earthquake and Megacities Inititative (EMI) and with the representatives of the institutions of Metro Manila for their participation in the review and test of the methodology in the city.

\section{References and Further Readings}

Barbat AH, Cardona OD (2003) Vulnerability and disaster risk indices from engineering perspective and holistic approach to consider hard and soft variables at urban level, IDB/ IDEA Program on Indicators for Disaster Risk Management, available in: http://idea. unalmzl.edu.co, National University of Colombia, Manizales.

Cardona OD (2001) Holistic evaluation of the seismic risk using complex dynamic systems (in Spanish), PhD Thesis, Technical University of Catalonia, Barcelona, Spain.

Cardona OD (2004) The need for rethinking the concepts of vulnerability and risk from a holistic perspective: a necessary review and criticism for effective risk management, in Bankoff G, Frerks G, Hilhorst D (eds.), Mapping Vulnerability: Disasters, Development and People, Earthscan Publishers, London, UK.

Cardona OD (2007) Disaster risk and vulnerability: Notions and metrics of environmental insecurity for a decision science, in Brauch HG, Oswald Spring U, Kameri-Mbote P, Mesjasz C, Grin J, Chourou B, Birkmann J (eds.), Coping with Global Environmental Change, Disasters and Security Threats, Challenges, Vulnerabilities and Risks,: SpringerVerlag (In press), The Netherlands.

Cardona OD, Barbat AH (2000) The Seismic risk and its prevention (in Spanish), Calidad Siderúrgica, Madrid, Spain.

Cardona OD, Hurtado JE (2000) Holistic seismic risk estimation of a metropolitan center, in Proceedings of 12th World Conference of Earthquake Engineering, Auckland, New Zealand.

Carreño ML (2006) Innovative techniques for seismic risk evaluation and risk management in urban centres: Ex-ante and ex-post actions (in Spanish), PhD Thesis, Technical University of Catalonia, Barcelona, Spain.

Carreño ML, Cardona OD, Barbat AH (2005a) System of Indicators for the risk evaluation (in Spanish), Monographs of Earthquake Engineering, IS-52, Barbat AH (ed.), International Center for Numerical Methods in Engineering (CIMNE), Barcelona, Spain..

Carreño ML, Cardona OD, Barbat AH (2005b) Seismic risk evaluation for an urban centre, Proceedings of the International Conference: 250th Anniversary of the 1755 Lisbon earthquake. Lisbon.

Carreño ML, Cardona OD Barbat AH (2007a) Urban Seismic Risk Evaluation: A Holistic Approach, Natural Hazards vol 40 num 1, pp 137:172, DOI 10.1007/s11069-006-0008-8.

Carreño ML, Cardona OD Barbat AH (2007b) A disaster risk management performance index, Natural Hazards vol 41 num 1, pp 1;20, DOI 10.1007/s11069-006-9008-y

EMI (2006) Megacity Indicators System MIS: Implementation in Metro Manila, $3 \mathrm{~cd}$ program of Earthquake and Megacities Inititative EMI, Pacific Disaster Center, ProVention Consortium.

ERN (2005) Seismic information system of Manizales - Risk (in Spanish), SISMAN + Riesgo, Evaluación de Riesgos Naturales ERN, National Planning Department, World Bank, Bogota,

ICC/CIMNE (2004) An Advanced Approach to Earthquake Risk Scenarios with Applications to Different European Towns, WP08, Application to Barcelona, RISK-UE Project.

Masure P (2003) Variables and indicators of vulnerability and disaster risk for land-use and urban or territorial planning, IDB/IDEA Program on Indicators for Disaster Risk Management, available in: http://idea.unalmzl.edu.co,National University of Colombia, Manizales. 
Saaty TL (2001), Decision making for leaders the analytic hierarchy process for decisions in a complex world, Pittsburgh RWS, USA.

Suarez DC (2007) Development of disaster risk and risk management indicators at urban level for diagnostic and planning in Manizales (in Spanish), MSc Thesis, National University of Colombia, Manizales.

UNDRO (1980) Natural Disasters and Vulnerability Analysis, Report of Experts Group Meeting, UNDRO, Geneva.

Universidad de los Andes (2005) Seismic risk and lossess scenarios of Bogotá (in Spanish), Centro de Estudios sobre Desastres y Riesgos CEDERI, Dirección de Prevención y Atención de Emergencias, DPAE, Bogotá, Colombia.

Zapata R (2004) personal communication in Washington. 